\title{
MAPPING DEGREE AND EULER CHARACTERISTIC
}

\author{
T. Fukui And A. KhovanskiI
}

\begin{abstract}
Let $V_{\delta}$ denote a local level surface for function-germ $f:\left(\mathbf{R}^{n+1}, 0\right) \rightarrow(\mathbf{R}, 0)$. A mapping degree formula for difference of the Euler characteristics of $V_{\delta} \cap\{g \leq 0\}$ and $V_{\delta} \cap\{g \geq 0\}$ is given, when level surfaces of a function $g:\left(\mathbf{R}^{n+1}, 0\right) \rightarrow(\mathbf{R}, 0)$ are parallelizable.
\end{abstract}

It is classically known that mapping degree is closely related to Euler characteristics. One of such relation is the following celebrated formula due to G. N. Khimshiashvili ([7]): Let $\left(x_{0}, x_{1}, \ldots, x_{n}\right)$ be a coordinate system of $\mathbf{R}^{n+1}$. Let $B_{\varepsilon}^{n+1}$ denote the open ball centered at $0 \in \mathbf{R}^{n+1}$ with radius $\varepsilon$. Let $f:\left(\mathbf{R}^{\varepsilon+1}, 0\right) \rightarrow(\mathbf{R}, 0)$ be an analytic function-germ and $V_{\delta}$ denote the local level surface of $f$, i.e.,

$$
V_{\delta}=B_{\varepsilon}^{n+1} \cap f^{-1}(\delta) \text { for } 0<|\delta| \ll \varepsilon \ll 1 .
$$

We denote its Euler characteristic by $\chi\left(V_{\delta}\right)$. Then the Khimshiashvili's formula asserts that, when $f$ defines an isolated singularity at 0 ,

$$
\operatorname{deg}(d f)=\operatorname{sign}(-\delta)^{n+1}\left(1-\chi\left(V_{\delta}\right)\right)
$$

where $d f$ is the map-germ defined by

$$
d f:\left(\mathbf{R}^{n+1}, 0\right) \rightarrow\left(\mathbf{R}^{n+1}, 0\right), \quad x \mapsto\left(f_{x_{0}}(x), f_{x_{1}}(x), \ldots, f_{x_{n}}(x)\right) .
$$

Here $f_{x_{i}}$ denote the partial derivative of $f$ by $x_{i}, i=0,1, \ldots, n$.

We consider a relative version of this formula. In [3], the first author considered the mapping degree of map-germs

$$
F:\left(\mathbf{R}^{n+1}, 0\right) \rightarrow\left(\mathbf{R}^{n+1}, 0\right), \quad x \mapsto\left(f(x), f_{x_{1}}(x), \ldots, f_{x_{n}}(x)\right)
$$

and showed that, if $F$ is finite, then

$$
\operatorname{deg}(F)=\operatorname{sign}(-\delta)^{n+1}\left(\chi\left(V_{\delta}\left(x_{0} \leq 0\right)\right)-\chi\left(V_{\delta}\left(x_{0} \geq 0\right)\right)\right.
$$

where $V_{\delta}\left(x_{0} \leq 0\right)=\left\{x \in V_{\delta}: x_{0} \leq 0\right\}$, and $V_{\delta}\left(x_{0} \geq 0\right)=\left\{x \in V_{\delta}: x_{0} \geq 0\right\}$.

In this paper, we consider an analytic function $g:\left(\mathbf{R}^{n+1}, 0\right) \rightarrow(\mathbf{R}, 0)$ so that there are $C^{\infty}$-vector fields $v_{1}, \ldots, v_{n}$ which span the tangent space of a level set

Received February 7, 2003; revised September 22, 2005. 
of $g$ at each regular point of $g$. We assume that $\nabla g, v_{1}, \ldots, v_{n}$ agree with the orientation of $\left(\mathbf{R}^{n+1}, 0\right)$ at each regular point of $g$ where $\nabla g$ is the gradient vector of $g$. We define a map $F$ by

$$
F:\left(\mathbf{R}^{n+1}, 0\right) \rightarrow\left(\mathbf{R}^{n+1}, 0\right), \quad x \mapsto\left(f(x), v_{1} f(x), \ldots, v_{n} f(x)\right) .
$$

The purpose is to show (Theorem 4.1) that, if $F$ is finite, and $V_{\delta} \cap \Sigma(g)=\emptyset$, then

$$
\operatorname{deg}(F)=\operatorname{sign}(-\delta)^{n+1}\left(\chi\left(V_{\delta}(g \leq 0)\right)-\chi\left(V_{\delta}(g \geq 0)\right)\right)
$$

where $V_{\delta}(g \leq 0)=\left\{x \in V_{\delta}: g(x) \leq 0\right\}$, and $V_{\delta}(g \geq 0)=\left\{x \in V_{\delta}: g(x) \geq 0\right\}$.

This formula will be proved in $\$ 4$ applying Morse theory to the restriction of $g$ to a level of $f$. In $\S 1$ we investigate the condition on the existence of such vector fields $v_{1}, \ldots, v_{n}$ and discuss explicit construction of them in some special case in $\$ 2$. Applying Morse theory to the restriction of $f$ to a level of $g$, we also show another topological interpretation of $\operatorname{deg} F$ in $\S 3$. In $\S 4$ we investigate the condition that $\left.g\right|_{V_{\delta}}$ is Morse and give a proof of $(0.1)$ and its variant.

In the last section, we consider a kind of 'product' of $d g$ and $d f$ and give a topological interpretation of its mapping degree. It is motivated by Remark 2.1 which is a consequence of the explicit form of $F$.

The authors thank to Professor T. Mizutani for letting them know about the use of Whitehead product. The authors also thank to the referee for his kind comments on the earlier version including pointing out some gramatical and typographic errors. Proposition 1.3 is inspired by the referee's comment.

\section{Condition (P) and the definition of the map $F$}

Let $L$ denote an oriented $(n+1)$-dimensional $C^{\infty}$-manifold and $g: L \rightarrow \mathbf{R}$ be a $C^{\infty}$-function on $L$. We fix a Riemannian metric on $L$ and denote the gradient of $g$ by $\nabla g$. We always consider the orientation of the set of regular points of the level set of $g$ so that $\nabla g$ and the orientation of the level set of $g$ agree with the orientation of $L$.

We consider the following condition on $g$.

$(\mathrm{P})$ : There exist $C^{\infty}$-vector fields $v_{1}(x), \ldots, v_{n}(x)$ on $L$ which span the tangent space of the level set of $g$ at a regular point $x$ of $g$, and the orientation of a level of $g$ there coincides with the orientation defined by $v_{1}(x), \ldots, v_{n}(x)$.

Definition 1.1. Let $g: L \rightarrow \mathbf{R}$ be a $C^{\infty}$-function with Condition (P). We define the map

$$
F: L \rightarrow \mathbf{R}^{n+1}, \quad \text { by } x \mapsto\left(f(x), v_{1} f(x), \ldots, v_{n} f(x)\right),
$$

where $f: L \rightarrow \mathbf{R}$ is a $C^{\infty}$-function.

In later sections, we investigate several topological interpretations of the mapping degree of $F$. In the rest of this section, we investigate Condition $(\mathrm{P})$ in general. 
1.1. Existence of vector fields $v_{1}, \ldots, v_{n}$ in Condition (P). We show the following

Proposition 1.2. Let $g:\left(\mathbf{R}^{n+1}, 0\right) \rightarrow(\mathbf{R}, 0)$ be a $C^{\infty}$-function which defines an isolated singularity at 0. Then, the following conditions are equivalent.

(i) There exist $C^{\infty}$-vector fields $v_{1}(x), \ldots, v_{n}(x)$ near 0 which span the tangent space of the level set of $g$ at a regular point $x$ of $g$.

(ii) One of the following conditions holds.

- $n=1,3,7$.

- $n$ is odd, $n \neq 1,3,7$, and $\operatorname{deg}(d g)$ is even.

- $n$ is even, and $\operatorname{deg}(d g)$ is zero.

First we consider more general set-up. Let $L$ be a manifold of dimension $n+1$, and let $g: L \rightarrow \mathbf{R}$ be a $C^{\infty}$-function. We denote $L^{\prime}=L-\Sigma(g)$, and assume that $L^{\prime}$ is parallelizable. Let $E$ denote the vector bundle on $L^{\prime}$ whose fiber is the tangent space of each level of $g$. We investigate the following

QUESTION. When $E$ is a trivial bundle?

If $E$ is $C^{0}$-trivial, then this bundle is $C^{\infty}$-trivial and there exist $C^{\infty}$-vector fields $w_{1}(x), \ldots, w_{n}(x)$ on $L^{\prime}$ which span $E$. Then $v_{i}(x)=b(x) w_{i}(x), i=1, \ldots, n$, satisfy Condition (P) where $b$ is a $C^{\infty}$-function on $L$ so that $\Sigma(g)=b^{-1}(0)$ and that $b$ is flat at $\Sigma(g)$, that is, all partial derivatives of order $k, k=0,1,2, \ldots$, vanish at each point of $\Sigma(g)$.

Since $L^{\prime}$ is parallelizable, there is an oriented orthonormal frame $\boldsymbol{e}_{0}, \boldsymbol{e}_{1}, \ldots, \boldsymbol{e}_{n}$ of the tangent bundle of $L^{\prime}$, and we can define the following Gauss map:

$$
\alpha: L^{\prime} \rightarrow S^{n}, \quad x \mapsto\left(a_{0}, a_{1}, \ldots, a_{n}\right) \quad \text { where } \frac{\nabla g}{\|\nabla g\|}=a_{0} \boldsymbol{e}_{0}+a_{1} \boldsymbol{e}_{1}+\cdots+a_{n} \boldsymbol{e}_{n}
$$

Let $\mathrm{SO}(n)$ denote the group of orthogonal $n \times n$ matrices with determinant 1 . Let us consider the map defined by

$$
p: \mathrm{SO}(n+1) \rightarrow S^{n}, \quad A \mapsto \text { the first column of } A .
$$

Proposition 1.3. Under the above assumption, the following conditions are equivalent.

(i) The vector bundle $E$ is $C^{0}$-trivial (and, thus $C^{\infty}$-trivial).

(ii) There is a continuous map $\beta: L^{\prime} \rightarrow S O(n+1)$ so that $\alpha=p \circ \beta$.

(iii) One of the following conditions holds.

- $n=1,3,7$.

- $n$ is odd, $n \neq 1,3,7$, and the induced map $\alpha_{\#}: \pi_{n}\left(L^{\prime}\right) \rightarrow \pi_{n}\left(S^{n}\right)$ is even.

- $n$ is even, and the induced map $\alpha_{\#}: \pi_{n}\left(L^{\prime}\right) \rightarrow \pi_{n}\left(S^{n}\right)$ is zero.

Here we say that a map $\alpha: G_{1} \rightarrow G_{2}$ between two abelian groups $G_{1}, G_{2}$ is even if for any $g_{1} \in G_{1}$ there is $g_{2} \in G_{2}$ with $f\left(g_{1}\right)=2 g_{2}$.

We say that a map $p: E \rightarrow B$ is a fibration in the sense of Serre if the 
following condition holds: for a CW complex $X$ and a homotopy $\alpha_{t}: X \rightarrow B$, $0 \leq t \leq 1$, if there is a map $\beta_{0}: X \rightarrow E$ with $p \circ \beta_{0}=\alpha_{0}$, then there is a homotopy $\beta_{t}: X \rightarrow E, 0 \leq t \leq 1$, with $p \circ \beta_{t}=\alpha_{t}$ for $0 \leq t \leq 1$. Serre.

We remark that the locally trivial fibration is a fibration in the sense of

Proof of Proposition 1.3. (i) $\Rightarrow$ (ii): If $E$ is trivial, then the associated $\mathrm{SO}(n)$-bundle with $E$ is trivial, and thus have non-zero section. This means (ii).

(ii) $\Rightarrow$ (i): If there is a continuous map $\beta: L^{\prime} \rightarrow \mathrm{SO}(n+1)$ so that $\alpha=p \circ \beta$, then there is an orthonormal frame which spans $E$, and we thus conclude that $E$ is trivial.

(ii) $\Rightarrow$ (iii): Since the map $p: \mathrm{SO}(n+1) \rightarrow S^{n}$ is a fibration with fiber $\mathrm{SO}(n)$, we have the following homotopy exact sequence:

$$
\pi_{n}(\mathrm{SO}(n+1)) \stackrel{p_{\#}}{\rightarrow} \pi_{n}\left(S^{n}\right) \rightarrow \pi_{n-1}(\mathrm{SO}(n)) \stackrel{l_{\#}}{\rightarrow} \pi_{n-1}(\mathrm{SO}(n+1)) \rightarrow 0
$$

where $l: \mathrm{SO}(n) \rightarrow \mathrm{SO}(n+1)$ denote an inclusion. Remark that the map $\beta: L^{\prime} \rightarrow$ $\mathrm{SO}(n+1)$ induces $\beta_{\#}: \pi_{n}\left(L^{\prime}\right) \rightarrow \pi_{n}(\mathrm{SO}(n+1))$ with $p_{\#} \circ \beta_{\#}=\alpha_{\#}$. Then the following fact (see [6, Chapter 8, Ex. 8]) implies (iii).

$$
\text { Kernel of } l_{\#}= \begin{cases}0, & \text { if } n=1,3,7 \\ \mathbf{Z} / 2 \mathbf{Z}, & \text { if } n \text { is odd and } n \neq 1,3,7 \\ \mathbf{Z}, & \text { if } n \text { is even. }\end{cases}
$$
theory.

(iii) $\Rightarrow$ (ii): We show this implication as an application of the obstruction

Let $S^{k}, k=0,1, \ldots, n-1$, be a $k$-dimensional sphere in $L^{\prime}$, and set $\alpha_{k}=\left.\alpha\right|_{S^{k}}$. The map $\alpha_{k}: S^{k} \rightarrow S^{n}$ represents the zero element of $\pi_{k}\left(S^{n}\right)$, since $\pi_{k}\left(S^{n}\right)=0$. Take a map $\beta_{k}^{\prime}: S^{k} \rightarrow \mathrm{SO}(n+1)$ which represents the zero element of $\pi_{k}(\operatorname{SO}(n+1))$. Since the map $p \circ \beta_{k}^{\prime}$ also represents zero of $\pi_{k}\left(S^{n}\right)$, there is a homotopy $\phi_{t}: S^{k} \rightarrow S^{n}, 0 \leq t \leq 1$, with $\phi_{0}=p \circ \beta_{k}^{\prime}$ and $\phi_{1}=\alpha_{k}$. Since $p: \mathrm{SO}(n+1) \rightarrow S^{n}$ is a fibration in the sense of Serre, there is a map $\beta_{k}: S^{k} \rightarrow$ $\mathrm{SO}(n+1)$ so that $p \circ \beta_{k}=\alpha_{k}$. If there is a $(k+1)$-dimensional ball $B^{k+1}$ in $L^{\prime}$ which bounds the sphere $S^{k}$ in $L^{\prime}$, then $\beta_{k}$ can be extended to $B^{k+1}$, since $\beta_{k}$ represents the zero in $\pi_{k}(\mathrm{SO}(n+1))$.

Let $S^{n}$ be an $n$-dimensional sphere in $L^{\prime}$ and set $\alpha_{n}=\left.\alpha\right|_{S^{n}}$. By (iii), the homotopy class of $\alpha_{n}$ is in the kernel of $l_{\#}$ in (1.2), because of (1.3). Since $p: \mathrm{SO}(n+1) \rightarrow S^{n}$ is a fibration in the sense of Serre, there is a map $\beta_{n}: S^{n} \rightarrow$ $\mathrm{SO}(n+1)$ so that $p \circ \beta_{n}=\alpha_{n}$.

Since $L^{\prime}$ is not compact, $L^{\prime}$ has a homotopy type of a CW complex of dimension $\leq n$, and we complete the proof.

Remark 1.4. Let $g_{1}:\left(\mathbf{R}^{n}, 0\right) \rightarrow(\mathbf{R}, 0)$ be a $C^{\infty}$-function. Let $U$ be a neighborhood of 0 and assume that $g_{1}$ is defined on $U$. Let $\pi: \mathbf{R}^{n+1} \rightarrow \mathbf{R}^{n}$ be a linear projection. Setting $g=g_{1} \circ \pi$ and $L=\pi^{-1}(U)$, we have $L^{\prime}=L-\Sigma(g) \simeq$ $\left(U-\Sigma\left(g_{1}\right)\right) \times \mathbf{R}$, that has a homotopy type of a CW complex of dimension $\leq$ 
$n-1$. By the above proof of the implication (iii) $\Rightarrow$ (ii), we conclude that $g$ satisfies Condition $(\mathrm{P})$.

Next we present two propositions which gives sufficient conditions for Condition $(\mathrm{P})$.

Proposition 1.5. Under the same assumption as Proposition 1.3, the vector bundle $E$ is trivial if there is a continuous map $\gamma: L^{\prime} \rightarrow P^{n}(\mathbf{R})$ so that $\varphi \circ \gamma$ is homotopic to $\alpha$ where $\varphi: P^{n}(\mathbf{R}) \rightarrow S^{n}$ is the map defined by

$$
[x]=\left[x_{0}: x_{1}: \cdots: x_{n}\right] \mapsto \frac{1}{S}\left(2 x_{0}^{2}-S, 2 x_{1} x_{0}, \ldots, 2 x_{n} x_{0}\right) \quad \text { where } S=\sum_{i=0}^{n} x_{i}^{2} .
$$

Let $q: S^{n} \rightarrow P^{n}(\mathbf{R})$ denote the map defined by $\left(x_{0}, x_{1}, \ldots, x_{n}\right) \mapsto$ $\left[x_{0}: x_{1}: \cdots: x_{n}\right]$. For a unit vector $x=\left(x_{0}, x_{1}, \ldots, x_{n}\right)$ and $y=\varphi \circ q(x)$, we see that $0, \boldsymbol{e}_{0}, x$, and $y$ are in the same plane and $2 \measuredangle \boldsymbol{e}_{0} 0 x=\measuredangle \boldsymbol{e}_{0} 0 y$. We remark that the map $\varphi$ is generically one-to-one and sends the set defined by $\left\{x_{0}=0\right\}$ to a point.

Proof. Let $x=\left(x_{0}, x_{1}, \ldots, x_{n}\right)$ be a non-zero vector in $\mathbf{R}^{n+1}$. Let $\psi_{x}$ : $\mathbf{R}^{n+1} \rightarrow \mathbf{R}^{n+1}$ denote the reflection sending the vector $x$ to $-x$. We remark that the map $\psi_{x}$ is represented by the matrix

$$
\left(\delta_{i, j}-\frac{2 x_{i} x_{j}}{S}\right)_{i, j=0,1, \ldots, n} \quad \text { where } \delta_{i, j}= \begin{cases}1, & \text { if } i=j \\ 0, & \text { otherwise }\end{cases}
$$

and the first column of the matrix for $\psi_{\boldsymbol{e}_{0}} \circ \psi_{x}$ represents the map $\varphi: P^{n}(\mathbf{R}) \rightarrow S^{n}$. Let $h_{t}$ denote a homotopy with $h_{0}=\varphi \circ \gamma$ and $h_{1}=\alpha$. We remark that there is a continuous map $\gamma_{1}: L^{\prime} \rightarrow \operatorname{SO}(n+1)$ with $\varphi=p \circ \gamma_{1}$. In fact, the map $\gamma_{1}=\psi \circ \gamma$ satisfy $\varphi=p \circ \gamma_{1}$ where $\psi: P^{n}(\mathbf{R}) \rightarrow \mathrm{SO}(n+1)$ is the embedding defined by $[x] \mapsto$ $\psi_{e_{0}} \circ \psi_{x}$. Since $p: \mathrm{SO}(n+1) \rightarrow S^{n}$ is a fibration in the sense of Serre, we obtain there is a continuous map $\alpha_{1}: L^{\prime} \rightarrow \operatorname{SO}(n+1)$ with $\alpha=p \circ \alpha_{1}$, and we complete the proof.

Proposition 1.6. Under the same assumption as Proposition 1.3, the vector bundle $E$ is trivial when one of the following conditions holds.

- $n$ is odd, and the induced map $\alpha^{*}: H^{n}\left(S^{n} ; \mathbf{Z}\right) \rightarrow H^{n}\left(L^{\prime} ; \mathbf{Z}\right)$ is even.

- $n$ is even, and the induced map $\alpha^{*}: H^{n}\left(S^{n} ; \mathbf{Z}\right) \rightarrow H^{n}\left(L^{\prime} ; \mathbf{Z}\right)$ is zero.

Proof. Assume first that $n$ is even and the induced map $\alpha^{*}: H^{n}\left(S^{n} ; \mathbf{Z}\right) \rightarrow$ $H^{n}\left(L^{\prime} ; \mathbf{Z}\right)$ is zero. Then, by Hopf's theorem (see [5, Chapter II, 8]) there is a homotopy $A: L^{\prime} \times[0,1] \rightarrow S^{n}, A(x, t)=\alpha_{t}(x)$, with the following properties:

- $\alpha_{0}=\alpha$.

- If $n$ is odd, then there are continuous maps $a: L^{\prime} \rightarrow S^{n}$ and $b: S^{n} \rightarrow S^{n}$ so that $b$ is of degree two and $\alpha=b \circ a$. We may assume that $b$ factors through the map $\varphi$. 
- If $n$ is even, then $\operatorname{Im} \alpha_{1}$ is a point.

Since $p: \mathrm{SO}(n+1) \rightarrow S^{n}$ is a fibration in the sense of Serre, we complete the proof as in the same way in the previous proposition.

Proof of Proposition 1.2. (i) $\Rightarrow$ (ii): If (i) holds, then (iii) of Proposition 1.3 holds, and (ii) holds.

(ii) $\Rightarrow$ (i): The implication (iii) $\Rightarrow$ (i) of Proposition 1.3 implies (ii) $\Rightarrow$ (i). The explicit construction of $v_{1}, \ldots, v_{n}$ in the next section gives another proof when $n=1,3,7$. When $n \neq 1,3,7$, Proposition 1.6 also gives another proof by Hopf's theorem (ibid.).

\section{Explicit construction of vector fields $v_{1}, \ldots, v_{n}$ in Condition (P)}

Let $g:\left(\mathbf{R}^{n+1}, 0\right) \rightarrow(\mathbf{R}, 0)$ be a polynomial (resp. analytic) function. Assume that one of the conditions in Proposition 1.3 (iii) (or in Proposition 1.2 (ii) when $g$ defines isolated singularity at 0 ) holds. Then are there polynomial (resp. analytic) vector fields $v_{1}, \ldots, v_{n}$ which span the tangent space of the level of $g$ at a regular point of $g$ ? The answer is affirmative if one of the following conditions holds.

(a) $n=1,3,7$.

(b) $g_{x_{0}}$ is not negative.

We are going to prove this assertion to construct vector field $v_{1}, \ldots, v_{n}$ explicitly. Let $L=\mathbf{R}^{n+1}$ and we denote by $\partial_{x_{i}}$ the unit vector $\boldsymbol{e}_{i}=(0, \ldots, \stackrel{i+1}{1}, \ldots, 0)$ for $i=0,1, \ldots, n$.

2.1. Case (a). If $n=1,3,7$, our explicit construction of the vector fields $v_{1}, \ldots, v_{n}$ is based on the multiplicative structure of complex, quotanion, Cayley numbers, respectively.

CASE $n=1$ : We consider the complex numbers $\mathbf{C}=\mathbf{R}+\mathbf{R} \boldsymbol{i}$ where $\boldsymbol{i}^{2}=-1$, and identify it with $\mathbf{R}^{2}$. Under this identification $\nabla g=g_{x_{0}}+g_{x_{1}} \boldsymbol{i}$. Then $i \nabla g=$ $-g_{x_{1}}+g_{x_{0}} i$ span the tangent space of the level set of $g$ at a regular point of $g$. In other words, the vector field $v_{1}$ in Condition $(\mathrm{P})$ is given by the following:

$$
v_{1}=i \nabla g=-g_{x_{1}} \partial_{x_{0}}+g_{x_{0}} \partial_{x_{1}} .
$$

CASE $n=3$ : We consider the quotanion numbers $Q=\mathbf{R}+\mathbf{R} \boldsymbol{i}+\mathbf{R} \boldsymbol{j}+\mathbf{R} \boldsymbol{k}$ with

$$
\boldsymbol{i}^{2}=\boldsymbol{j}^{2}=\boldsymbol{k}^{2}=-1, \quad \boldsymbol{i} \boldsymbol{j}=-\boldsymbol{j i}=\boldsymbol{k}, \quad \boldsymbol{j} \boldsymbol{k}=-\boldsymbol{k} \boldsymbol{j}=\boldsymbol{i}, \quad \boldsymbol{k} \boldsymbol{i}=-\boldsymbol{i} \boldsymbol{k}=\boldsymbol{j} .
$$

We set $\bar{x}=a_{0}-a_{1} \boldsymbol{i}-a_{2} \boldsymbol{j}-a_{3} \boldsymbol{k}$ when $x=a_{0}+a_{1} \boldsymbol{i}+a_{2} \boldsymbol{j}+a_{3} \boldsymbol{k}$. Since $x \bar{x}=$ $\sum_{i=0}^{3} a_{i}^{2}, x$ has the inverse $\bar{x} /(x \bar{x})$ if $x \neq 0$. We identify $Q$ with $\mathbf{R}^{4}$. We remark that $\langle x, y\rangle:=\operatorname{Re}(x \bar{y}) \quad(x, y \in Q)$ is the Euclidean inner product of $\mathbf{R}^{4}$. Under this identification we have that $\nabla g=g_{x_{0}}+g_{x_{1}} \boldsymbol{i}+g_{x_{2}} \boldsymbol{j}+g_{x_{3}} \boldsymbol{k}$. Since $(1, \boldsymbol{i}, \boldsymbol{j}, \boldsymbol{k})$ forms an orthonormal frame of the tangent space of $\mathbf{R}^{4},(\nabla g, i \nabla g, j \nabla g, \boldsymbol{k} \nabla g)$ 
forms also an orthogonal frame of the tangent space of $\mathbf{R}^{4}$, when $\nabla g \neq 0$. This implies that $i \nabla g, j \nabla g, k \nabla g$ span the tangent space of the level set of $g$ at a regular point of $g$. In other words, the vector fields $v_{1}, v_{2}, v_{3}$ in Condition $(\mathrm{P})$ are given by the following:

$$
\begin{aligned}
& v_{1}=\boldsymbol{i} \nabla g=-g_{x_{1}} \partial_{x_{0}}+g_{x_{0}} \partial_{x_{1}}-g_{x_{3}} \partial_{x_{2}}+g_{x_{2}} \partial_{x_{3}}, \\
& v_{2}=\boldsymbol{j} \nabla g=-g_{x_{2}} \partial_{x_{0}}+g_{x_{3}} \partial_{x_{1}}+g_{x_{0}} \partial_{x_{2}}-g_{x_{1}} \partial_{x_{3}}, \\
& v_{3}=\boldsymbol{k} \nabla g=-g_{x_{3}} \partial_{x_{0}}-g_{x_{2}} \partial_{x_{1}}+g_{x_{1}} \partial_{x_{2}}+g_{x_{0}} \partial_{x_{3}} .
\end{aligned}
$$

CASE $n=7$ : We consider Cayley numbers $\mathfrak{C}=Q+Q e$ with

$$
(q+r e)(s+t e)=(q s-\bar{t} r)+(t q+r \bar{s}) e, \quad q, r, s, t \in Q .
$$

We set $\bar{x}=\bar{q}-r e$ when $x=q+r e$. Since $x \bar{x}=q \bar{q}+r \bar{r}, x$ has the inverse $\bar{x} /(x \bar{x})$ if $x \neq 0$. We identify $\mathfrak{C}$ with $\mathbf{R}^{8}$ and remark that $\langle x, y\rangle:=\operatorname{Re}(x \bar{y})(x, y \in \mathfrak{C})$ is the Euclidean inner product of $\mathbf{R}^{8}$. Under this identification we have that $\nabla g=$ $g_{x_{0}}+g_{x_{1}} \boldsymbol{i}+g_{x_{2}} \boldsymbol{j}+g_{x_{3}} \boldsymbol{k}+\left(g_{x_{4}}+g_{x_{5}} \boldsymbol{i}+g_{x_{6}} \boldsymbol{j}+g_{x_{7}} \boldsymbol{k}\right) e$. Then $\boldsymbol{i} \nabla g, \boldsymbol{j} \nabla g, \boldsymbol{k} \nabla g, e \nabla g$, $\boldsymbol{i} e \nabla g, \boldsymbol{j} e \nabla g, \boldsymbol{k} e \nabla g$ span the tangent space of the level set of $g$ at a regular point of $g$. In other words, the vector fields $v_{1}, \ldots, v_{7}$ in Condition $(\mathrm{P})$ are given by the following:

$$
\begin{gathered}
v_{1}=\boldsymbol{i} \nabla g=-g_{x_{1}} \partial_{x_{0}}+g_{x_{0}} \partial_{x_{1}}-g_{x_{3}} \partial_{x_{2}}+g_{x_{2}} \partial_{x_{3}}-g_{x_{5}} \partial_{x_{4}}+g_{x_{4}} \partial_{x_{5}}+g_{x_{7}} \partial_{x_{6}}-g_{x_{6}} \partial_{x_{7}}, \\
v_{2}=\boldsymbol{j} \nabla g=-g_{x_{2}} \partial_{x_{0}}+g_{x_{3}} \partial_{x_{1}}+g_{x_{0}} \partial_{x_{2}}-g_{x_{1}} \partial_{x_{3}}-g_{x_{6}} \partial_{x_{4}}-g_{x_{7}} \partial_{x_{5}}+g_{x_{4}} \partial_{x_{6}}+g_{x_{5}} \partial_{x_{7}}, \\
v_{3}=\boldsymbol{k} \nabla g=-g_{x_{3}} \partial_{x_{0}}-g_{x_{2}} \partial_{x_{1}}+g_{x_{1}} \partial_{x_{2}}+g_{x_{0}} \partial_{x_{3}}-g_{x_{7}} \partial_{x_{4}}+g_{x_{6}} \partial_{x_{5}}-g_{x_{5}} \partial_{x_{6}}+g_{x_{4}} \partial_{x_{7}}, \\
v_{4}=e \nabla g=-g_{x_{4}} \partial_{x_{0}}+g_{x_{5}} \partial_{x_{1}}+g_{x_{6}} \partial_{x_{2}}+g_{x_{7}} \partial_{x_{3}}+g_{x_{0}} \partial_{x_{4}}-g_{x_{1}} \partial_{x_{5}}-g_{x_{2}} \partial_{x_{6}}-g_{x_{3}} \partial_{x_{7}}, \\
v_{5}=\boldsymbol{i} e \nabla g=-g_{x_{5}} \partial_{x_{0}}-g_{x_{4}} \partial_{x_{1}}+g_{x_{7}} \partial_{x_{2}}-g_{x_{6}} \partial_{x_{3}}+g_{x_{1}} \partial_{x_{4}}+g_{x_{0}} \partial_{x_{5}}+g_{x_{3}} \partial_{x_{6}}-g_{x_{2}} \partial_{x_{7}}, \\
v_{6}=\boldsymbol{j} e \nabla g=-g_{x_{6}} \partial_{x_{0}}-g_{x_{7}} \partial_{x_{1}}-g_{x_{4}} \partial_{x_{2}}+g_{x_{5}} \partial_{x_{3}}+g_{x_{2}} \partial_{x_{4}}-g_{x_{3}} \partial_{x_{5}}+g_{x_{0}} \partial_{x_{6}}+g_{x_{1}} \partial_{x_{7}}, \\
v_{7}=\boldsymbol{k} e \nabla g=-g_{x_{7}} \partial_{x_{0}}+g_{x_{6}} \partial_{x_{1}}-g_{x_{5}} \partial_{x_{2}}-g_{x_{4}} \partial_{x_{3}}+g_{x_{3}} \partial_{x_{4}}+g_{x_{2}} \partial_{x_{5}}-g_{x_{1}} \partial_{x_{6}}+g_{x_{0}} \partial_{x_{7}} .
\end{gathered}
$$

Remark 2.1. In the above construction, the map $F$ (Definition 1.1) coincides with

$$
p(\overline{\nabla g}, \nabla f): \mathbf{R}^{n+1} \rightarrow \mathbf{R}^{n+1}, \quad x \mapsto p(\overline{\nabla g(x)}, \nabla f(x))
$$

except the first component, where $p: \mathbf{R}^{n+1} \times \mathbf{R}^{n+1} \rightarrow \mathbf{R}^{n+1}$ is the product of the complex, quotanion, Cayley numbers, respectively. In fact, the $\boldsymbol{e}_{i}$ component, $i=1, \ldots, n$, of $F$ is $\left\langle\boldsymbol{e}_{i} \nabla g, \nabla f\right\rangle=\operatorname{Re}\left(p\left(-\boldsymbol{e}_{i} \overline{\nabla g}, \nabla f\right)\right)=\operatorname{Re}\left(p\left(-\boldsymbol{e}_{i}, p(\overline{\nabla g}, \nabla f)\right)\right.$, which is the $\boldsymbol{e}_{i}$ component of $p((\overline{\nabla g}, \nabla f))$. Here we use the fact $\operatorname{Re}((a b) c)=\operatorname{Re}(a(b c))$ for any complex, quotanion, Cayley numbers $a, b, c$, respectively.

2.2. Case (b). Assume that $g_{x_{0}}$ is not negative. This means that the mapping degree of $d g$ is zero. We define vector fields $v_{i}, i=1, \ldots, n$, by 


$$
v_{i}=g_{x_{i}} \partial_{x_{0}}+\sum_{j=1}^{n}\left(g_{x_{i}} g_{x_{j}}-\delta_{i, j} T\right) \partial_{x_{j}} \quad \text { where } T=g_{x_{0}}+\sum_{j=1}^{n} g_{x_{j}}^{2} .
$$

Then $v_{1}, \ldots, v_{n}$ span the tangent space of each level of $g$ at each regular point of $g$.

It is clear that these $v_{1}, \ldots, v_{n}$ are polynomial (resp. analytic) vector fields when $g$ is a polynomial (resp. analytic).

Proof. It is easy to see that $\left\langle\nabla g, v_{i}\right\rangle=0$ for $i=1, \ldots, n$. So it is enough to show that $\nabla g, v_{1}, \ldots, v_{n}$ are linearly independent on $\mathbf{R}^{n}-\Sigma(g)$. The coefficient matrix of vector fields $\nabla g, v_{1}, \ldots, v_{n}$ is

$$
M=\left(\begin{array}{cc}
g_{x_{0}} & g_{x_{i}} \\
g_{x_{j}} & g_{x_{i}} g_{x_{j}}-\delta_{i, j} T
\end{array}\right)_{i, j=1, \ldots, n}
$$

and its determinant is $T^{n-1} \sum_{i=0}^{n} g_{x_{i}}^{2}$. This implies that $\nabla g, v_{1}, \ldots, v_{n}$ are linearly dependent only on $\{T=0\} \cup \Sigma(g)$. By assumption $\{T=0\} \cup \Sigma(g)=\Sigma(g)$, and we are done.

Remark that $M \boldsymbol{e}_{0}=\nabla g, M \nabla g=\|\nabla g\|^{2} \boldsymbol{e}_{0}$, and $M v=-T v$ when $\left\langle v, \boldsymbol{e}_{0}\right\rangle=$ $\langle v, \nabla g\rangle=0$.

Remark 2.2. The matrix appeared in the proof of Proposition 1.5 suggests another explicit construction of the vector field $v_{1}, \ldots, v_{n}$ in some special case. Let us find an $x$ with $\varphi(x)=\nabla g /\|\nabla g\|$ where $\nabla g$ denotes the gradient of $g$. Looking the first component, we have $\frac{2 x_{0}^{2}}{S}-1=\frac{g_{x_{0}}}{\|\nabla g\|}$ and

$$
\left(1-\frac{g_{x_{0}}}{\|\nabla g\|}\right) x_{0}^{2}=\left(x_{1}^{2}+\cdots+x_{n}^{2}\right)\left(1+\frac{g_{x_{0}}}{\|\nabla g\|}\right) .
$$

We then obtain

$$
\begin{aligned}
\left(\frac{x_{0}}{1+\frac{g_{x_{0}}}{\|\nabla g\|}}\right)^{2} & =\frac{x_{1}^{2}+\cdots+x_{n}^{2}}{\left(\frac{g_{x_{1}}}{\|\nabla g\|}\right)^{2}+\cdots+\left(\frac{g_{x_{n}}}{\|\nabla g\|}\right)^{2}} \\
& =\frac{x_{1}^{2}+\cdots+x_{n}^{2}}{\left(\frac{2 x_{1} x_{0}}{S}\right)^{2}+\cdots+\left(\frac{2 x_{n} x_{0}}{S}\right)^{2}}=\left(\frac{S}{2 x_{0}}\right)^{2} .
\end{aligned}
$$

We thus conclude

$$
\left(x_{0}, x_{1}, \ldots, x_{n}\right)=k\left(\nabla g \pm\|\nabla g\| \boldsymbol{e}_{0}\right) \quad \text { where } k=\frac{S}{2 x_{0}\|\nabla g\|} .
$$


Choosing the sign + , and setting $k=1$, we have

$$
v_{i}:=\varphi\left(\boldsymbol{e}_{i}\right)=\frac{1}{\|\nabla g\|}\left(g_{x_{0}} \partial_{x_{0}}+\sum_{j=1}^{n}\left(\frac{g_{x_{i}} g_{x_{j}}}{\|\nabla g\|+g_{x_{0}}}+\delta_{i, j}\|\nabla g\|\right) \partial_{x_{j}}\right), \quad i=1, \ldots, n .
$$

They are the desired vector fields which make sense whenever $\nabla g+\|\nabla g\| \boldsymbol{e}_{0} \neq 0$. Remark that the last condition implies the mapping degree of $d g$ is zero. But, in this construction, it is not clear that $v_{1}, \ldots, v_{n}$ are polynomial (resp. analytic) vector fields when $g$ is a polynomial (resp. analytic).

\section{Restricting $f$ to the level of $g$}

THEOREM 3.1. Let $L$ be a $C^{\infty}$-manifold of dimension $n+1$ and $f, g: L \rightarrow \mathbf{R}$ $C^{\infty}$-functions. We assume that 0 is a regular value of $g: L \rightarrow \mathbf{R}$ and set $N=$ $g^{-1}(0)$. We assume that $g$ satisfies Condition $(P)$ and the map

$$
\bar{F}: N \rightarrow S^{n}, \quad x \mapsto \frac{\left(f(x), v_{1} f(x), \ldots, v_{n} f(x)\right)}{\left\|\left(f(x), v_{1} f(x), \ldots, v_{n} f(x)\right)\right\|},
$$

is well-defined and finite.

(i) If $L_{+}=\{x \in L: f(x) \geq 0\}$ is compact, then

$$
\operatorname{deg} \bar{F}=\chi(N(f \geq 0), N(f=0))
$$

where $N(f \geq 0)$ denotes the set $\{x \in N: f(x) \geq 0\}$, and so on.

(ii) If $L_{-}=\{x \in L: f(x) \leq 0\}$ is compact, then we obtain

$$
\operatorname{deg} \bar{F}=(-1)^{n+1} \chi(N(f \leq 0), N(f=0)) .
$$

Proof. Take the point $(1,0, \ldots, 0)$ and consider its preimage by $\bar{F}$. They are the critical points of $f: N \rightarrow \mathbf{R}$ in the region $\{f>0\}$. If $\left.f\right|_{N}$ is Morse (we can assume this after small perturbation of $f$ if necessary), we obtain

$$
\operatorname{Hess}\left(\left.f\right|_{N}\right)(x)=\frac{\partial \bar{F}}{\partial y}(x),
$$

where $y$ denotes an oriented coordinate system of $N$. This implies the first equality.

Next take the point $(-1,0, \ldots, 0)$ and apply the similar discussion for $-f$ on the region $\{f \leq 0\}$. We then obtain the second equality. $\operatorname{deg} F_{0}$.

When $F$ induces a finite map germ $F_{0}:\left(L, F^{-1}(0)\right) \rightarrow\left(\mathbf{R}^{n+1}, 0\right), \operatorname{deg} \bar{F}=$

Remark 3.2. Assume that $L$ is compact. If $n$ is odd, we have that $\operatorname{deg} \bar{F}=\frac{1}{2} \chi(N(f=0))$ and $\chi(N(f \geq 0))=\chi(N(f \leq 0))$. We consider the following Gauss map 


$$
G: N(f=0) \rightarrow S^{n-1}, \quad x \mapsto \frac{\left(v_{1} f(x), \ldots, v_{n} f(x)\right)}{\left\|\left(v_{1} f(x), \ldots, v_{n} f(x)\right)\right\|} .
$$

Using the fact stated in $[8, \S 6]$, we obtain that the degree of this Gauss map is equal to the sum of indices of $\nabla f$ in $N(f \geq 0)$, which is equal to $\operatorname{deg} \bar{F}$. So we conclude that $\operatorname{deg} G=\frac{1}{2} \chi(N(f=0))$.

\section{Restricting $g$ to the level of $f$}

THeOREM 4.1. Let $f, g: B_{\varepsilon}^{n+1} \rightarrow \mathbf{R}$ be analytic functions with $f(0)=$ $g(0)=0$. We assume that the singular set of $(f, g)$, which is defined by

$$
X=\left\{x \in B_{\varepsilon}^{n+1}: \operatorname{rank}\left(\begin{array}{llll}
f_{x_{0}}(x) & f_{x_{1}}(x) & \cdots & f_{x_{n}}(x) \\
g_{x_{0}}(x) & g_{x_{1}}(x) & \cdots & g_{x_{n}}(x)
\end{array}\right)<2\right\},
$$

is of dimension 1 . We choose $\varepsilon>0$ small enough so that

- the number of connected components of $(X-\{0\}) \cap B_{\varepsilon^{\prime}}^{n+1}$ does not change if $0<\varepsilon^{\prime} \leq \varepsilon$, and

- the functions $f$ and $g$ do not change the sign on each connected component of $X-\{0\}$.

We choose $\delta$, a regular value of $f$, which is close enough to 0 , and set $V_{\delta}=$ $\left\{x \in B_{\varepsilon}^{n+1}: f(x)=\delta\right\}$. We assume that $g$ satisfies Condition $(P)$. If $V_{\delta} \cap \Sigma(g)$ $=\emptyset,\left.g\right|_{V_{\delta}}$ is a Morse function, and the map-germ

$$
F:\left(\mathbf{R}^{n+1}, 0\right) \rightarrow\left(\mathbf{R}^{n+1}, 0\right), \quad x \mapsto\left(f(x), v_{1} f(x), \ldots, v_{n} f(x)\right),
$$

is finite, then we have the following:

$$
\begin{aligned}
\operatorname{deg}(F) & =\operatorname{sign}(-\delta)^{n+1}\left(\chi\left(V_{\delta}(g \leq 0)\right)-\chi\left(V_{\delta}(g \geq 0)\right)\right) \\
& =\operatorname{sign}(-\delta)^{n+1}\left(\chi\left(\bar{V}_{\operatorname{sign}(\delta)-}\right)-\chi\left(\bar{V}_{\operatorname{sign}(\delta)+}\right)\right)
\end{aligned}
$$

Here we denote by $V_{\delta}(g \leq 0)$ the set $\left\{x \in V_{\delta}: g(x) \leq 0\right\}$, and so on. We also denote by $\bar{V}_{\operatorname{sign}(\delta) \pm}$ the set $\left\{x \in S_{\varepsilon}^{n}: \operatorname{sign}(\delta) f(x) \geq 0, \pm g(x) \geq 0\right\}$ for $0<\varepsilon \ll 1$.

Remark 4.2. Consider the jet space $J=J^{1}\left(\mathbf{R}^{n+1}, \mathbf{R}^{2}\right)$ with coordinates

$$
\left(x_{0}, x_{1} \ldots, x_{n}, y, z, p_{0}, p_{1}, \ldots, p_{n}, q_{0}, q_{1}, \ldots, q_{n}\right),
$$

so that the jet section of a map $(f, g): \mathbf{R}^{n+1} \rightarrow \mathbf{R}^{2}$ is defined by

$$
y=f(x), \quad p_{i}=f_{x_{i}}(x), \quad z=g(x), \quad q_{i}=g_{x_{i}}(x), \quad i=0,1, \ldots, n .
$$

Let $\Sigma_{i}, i=0,1,2$, be the submanifolds of the jet space $J$ defined by

$$
\operatorname{rank}\left(\begin{array}{cccc}
p_{0} & p_{1} & \cdots & p_{n} \\
q_{0} & q_{1} & \cdots & q_{n}
\end{array}\right)=i .
$$

If the map $(f, g):\left(\mathbf{R}^{n+1}, 0\right) \rightarrow\left(\mathbf{R}^{2}, 0\right)$ is transverse to $\Sigma_{0}, \quad \Sigma_{1}$ and $\Sigma_{2}$ on $\left(\mathbf{R}^{n+1}-0,0\right)$, then the singular set $X$ of $(f, g)$ is of dimension 1 , and the num- 
ber of connected components of $(X-\{0\}) \cap B_{\varepsilon^{\prime}}^{n+1}$ does not change if $0<\varepsilon^{\prime} \ll 1$. This means that the condition on $X$ is a generic condition, if $\varepsilon>0$ is small enough.

Lemma 4.3. Let $\gamma:(\mathbf{R}, 0) \rightarrow(X, 0)$ be a $C^{\infty}$-map with $f \circ \gamma(t) \neq 0$ when $0<|t| \ll 1$. We then obtain $\nabla f(\gamma(t))$ is not identically zero. Since $\gamma(t) \in X$, there are real numbers $\lambda(t)$ so that $\nabla g(\gamma(t))=\lambda(t) \nabla f(\gamma(t))$, when $0<|t| \ll 1$.

(i) If $g \circ \gamma(t)$ is identically zero, then $\lambda(t)$ is also identically zero.

(ii) If $g \circ \gamma(t)$ is not identically zero, then $\operatorname{sign} \lambda=\operatorname{sign}(g / f)$ along $\gamma(t), 0<$ $|t| \ll 1$.

Proof. Assume that $\nabla f(\gamma(t))$ is identically zero. We then have

$$
\frac{d}{d t} f \circ \gamma(t)=\sum_{i=0}^{n} \frac{\partial f}{\partial x_{i}}(\gamma(t)) \frac{d}{d t}\left(x_{i} \circ \gamma(t)\right) \equiv 0,
$$

which implies $f \circ \gamma(t)$ is constant. This shows the first assertion. If $g \circ \gamma(t)$ is identically zero, then

$$
\begin{aligned}
0=\frac{d}{d t}(f \circ \gamma(t) \cdot g \circ \gamma(t)) & =g \circ \gamma(t) \frac{d}{d t} f \circ \gamma(t)+f \circ \gamma(t) \frac{d}{d t} g \circ \gamma(t) \\
& =\lambda(t) f \circ \gamma(t) \frac{d}{d t} f \circ \gamma(t)
\end{aligned}
$$

and we conclude $\lambda(t)$ is identically zero. This completes the proof of (i). The assertion (ii) is a consequence of Cauchy's mean value theorem.

Take a point $x \in X-\{0\}$.

- If $\delta=f(x)$ is a regular value of $f$, then $x$ is a critical point of $\left.g\right|_{\{f=\delta\}}$.

- If $\delta^{\prime}=g(x)$ is a regular value of $g$, then $x$ is a critical point of $\left.f\right|_{\left\{g=\delta^{\prime}\right\}}$. The following lemma clarifies when $\left.g\right|_{V_{\delta}}$ is a Morse function.

LEMMA 4.4. Let $\delta$ be a regular value of $f$. For $x \in X \cap V_{\delta}$ there exists a real number $\lambda$ so that $\nabla g(x)=\lambda \nabla f(x)$. Then $\left.g\right|_{V_{\delta}}$ is Morse at $x$, if and only if

$$
\left|\begin{array}{cc}
0 & f_{x_{j}} \\
f_{x_{i}} & g_{x_{i} x_{j}}-\lambda f_{x_{i} x_{j}}
\end{array}\right|_{i, j=0,1, \ldots, n} \neq 0 \text { at } x .
$$

Proof. It is enough to prove the lemma assuming $f_{x_{0}}(x) \neq 0$. Then there is a function $\varphi\left(x_{1}, \ldots, x_{n}\right)$ with

$$
f\left(\varphi\left(x_{1}, \ldots, x_{n}\right), x_{1}, \ldots, x_{n}\right) \equiv \delta .
$$

Differentiating (4.4) by $x_{i}, i=1, \ldots, n$, we obtain

$$
f_{x_{0}} \varphi_{x_{i}}+f_{x_{i}} \equiv 0,
$$


and $\varphi_{x_{i}}=-f_{x_{0}}^{-1} f_{x_{i}}$. Differentiating (4.5) by $x_{j}, j=1, \ldots, n$, we obtain that

$$
f_{x_{0} x_{0}} \varphi_{x_{i}} \varphi_{x_{j}}+f_{x_{0} x_{j}} \varphi_{x_{i}}+f_{x_{0} x_{i}} \varphi_{x_{j}}+f_{x_{i} x_{j}}+f_{x_{0}} \varphi_{x_{i} x_{j}} \equiv 0 .
$$

We consider the Hessian of the function $G\left(x_{1}, \ldots, x_{n}\right):=g\left(\varphi\left(x_{1}, \ldots, x_{n}\right), x_{1}, \ldots, x_{n}\right)$ at its critical point $x$. Similar computation shows that $G_{x_{i}}=g_{x_{0}} \varphi_{x_{i}}+g_{x_{i}}, i=$ $1, \ldots, n$, and $\lambda=g_{x_{0}} f_{x_{0}}^{-1}$ at $x \in X$. We also obtain that

$$
\begin{aligned}
G_{x_{i} x_{j}} & =g_{x_{0} x_{0}} \varphi_{x_{i}} \varphi_{x_{j}}+g_{x_{0} x_{j}} \varphi_{x_{i}}+g_{x_{0} x_{i}} \varphi_{x_{j}}+g_{x_{i} x_{j}}+g_{x_{0}} \varphi_{x_{i}, x_{j}} \\
& =\left(g_{x_{0} x_{0}}-\lambda f_{x_{0} x_{0}}\right) \varphi_{x_{i}} \varphi_{x_{j}}+\left(g_{x_{0} x_{j}}-\lambda f_{x_{0} x_{j}}\right) \varphi_{x_{i}}+\left(g_{x_{0} x_{i}}-\lambda f_{x_{0} x_{i}}\right) \varphi_{x_{j}}+\left(g_{x_{i} x_{j}}-\lambda f_{x_{i} x_{j}}\right)
\end{aligned}
$$

at $x$ by (4.6). Therefore we conclude that

$$
\begin{aligned}
\operatorname{det}\left(G_{x_{i} x_{j}}\right)_{i, j=1, \ldots, n} & =\left|\begin{array}{cccc}
-1 & 0 & 0 & 0 \\
0 & 0 & -1 & 0 \\
0 & -1 & 0 & 0 \\
0 & 0 & 0 & G_{x_{i} x_{j}}
\end{array}\right|_{i, j=1, \ldots, n} \\
& =\left|\begin{array}{cccc}
-1 & 0 & 0 & \left(g_{x_{0} x_{0}}-\lambda f_{x_{0} x_{0}}\right) \varphi_{x_{j}} \\
0 & 0 & -1 & \varphi_{x_{j}} \\
0 & -1 & 0 & g_{x_{0} x_{j}}-\lambda f_{x_{0} x_{j}} \\
\varphi_{x_{i}} & \varphi_{x_{i}} & g_{x_{0} x_{i}}-\lambda f_{x_{0} x_{i}} & g_{x_{i} x_{j}}-\lambda f_{x_{i} x_{j}} \\
-1 & 0 & g_{x_{0} x_{0}}-\lambda f_{x_{0} x_{0}} & 0 \\
0 & 0 & -1 & \varphi_{x_{j}} \\
1 & -1 & 0 & g_{x_{0} x_{j}}-\lambda f_{x_{0} x_{j}} \\
0 & \varphi_{x_{i}} & g_{x_{0} x_{i}}-\lambda f_{x_{0} x_{i}} & g_{x_{i} x_{j}}-\lambda f_{x_{i} x_{j}}
\end{array}\right|_{i, j=1, \ldots, n} \\
& =\left|\begin{array}{cccc}
-1 & 0 & g_{x_{0} x_{0}}-\lambda f_{x_{0} x_{0}} & 0 \\
0 & 0 & -1 & \varphi_{x_{j}} \\
0 & -1 & g_{x_{0} x_{0}}-\lambda f_{x_{0} x_{0}} & g_{x_{0} x_{j}}-\lambda f_{x_{0} x_{j}} \\
0 & \varphi_{x_{i}} & g_{x_{0} x_{i}}-\lambda f_{x_{0} x_{i}} & g_{x_{i} x_{j}}-\lambda f_{x_{i} x_{j}}
\end{array}\right|_{i, j=1, \ldots, n} \\
& =-f_{x_{0}}^{-2}\left|\begin{array}{ccc}
0 & f_{x_{j}} & g_{x_{i} x_{j}}-\lambda f_{x_{i} x_{j}}
\end{array}\right|_{i, j=0,1, \ldots, n}
\end{aligned}
$$

at $x$, which completes the proof.

If $x$ is a regular point of $f$ and $g$, then we have

Lemma 4.5. $\operatorname{sign} \operatorname{Hess}\left(\left.f\right|_{\left\{g=\delta^{\prime}\right\}}\right)=\operatorname{sign}\left((-\lambda)^{n} \operatorname{Hess}\left(\left.g\right|_{\{f=\delta\}}\right)\right)$ at $x \in X$ near 0 .

Proof. Since $x$ is a regular point of $g$, there exists a coordinate system $\left(x_{0}, x_{1}, \ldots, x_{n}\right)$ centered at $x$ so that $x_{0}=g(x)$. We consider $f$ as a functions 
of $\left(x_{0}, x_{1}, \ldots, x_{n}\right)$ and write $f=f\left(x_{0}, x_{1}, \ldots, x_{n}\right)$. By implicit function theorem, there exists a $C^{\infty}$-function $\psi\left(x_{1}, \ldots, x_{n}\right)$ so that $f\left(\psi\left(x_{1}, \ldots, x_{n}\right), x_{1}, \ldots, x_{n}\right)=\delta$. Then we obtain that

$$
\begin{aligned}
& f_{x_{0}} \psi_{x_{i}}+f_{x_{i}}=0, \quad \text { for } i=1, \ldots, n, \quad \text { and } \\
& f_{x_{0}} \psi_{x_{i} x_{j}}+f_{x_{i} x_{j}} \equiv 0 \bmod \psi_{x_{i}}, \quad \text { for } i, j=1, \ldots, n
\end{aligned}
$$

This means $f_{x_{i} x_{j}}(x)=-f_{x_{0}} \psi_{x_{i} x_{j}}(x)$, which implies the lemma.

Proof of Theorem 4.1. We choose a non-zero number $\delta$ close enough to 0 so that the numbers of connected components of $\{x \in X: 0<\operatorname{sign}(\delta) f(x)<\varepsilon\}$ do not depend on $\varepsilon$ with $0<\varepsilon<|\delta|$. Let $\alpha(\varepsilon), 0<\operatorname{sign}(\delta) \varepsilon<|\delta|$, denote the halfbranch of $X$ which contains $x$. We assume that $f(\alpha(\varepsilon))=\varepsilon$. We extend the function $\varepsilon$ to a neighborhood of $X$ near $x$ and denote it by the same letter $\varepsilon$. We consider functions $g_{1}, \ldots, g_{n}$ so that $X=\left\{g_{1}=\cdots=g_{n}=0\right\}$ near $x$ and so that $\nabla g_{i}(x)=v_{i}(x)$ for $i=1, \ldots, n$. We see that $\left(\varepsilon, g_{1}, \ldots, g_{n}\right)$ and $\left(g, g_{1}, \ldots, g_{n}\right)$ are systems of coordinates near $x$. Then we obtain that

$$
\frac{\partial F}{\partial\left(g, g_{1}, \ldots, g_{n}\right)}=\frac{\partial\left(\varepsilon, g_{1}, \ldots, g_{n}\right)}{\partial\left(g, g_{1}, \ldots, g_{n}\right)} \frac{\partial F}{\partial\left(\varepsilon, g_{1}, \ldots, g_{n}\right)}=\lambda^{-1}\left|\begin{array}{cc}
1 & * \\
0 & v_{i} v_{j} f
\end{array}\right| \quad \text { at } x,
$$

since $v_{i} f(\alpha(\varepsilon))=0$ and $\langle\dot{\alpha}, \nabla f\rangle=1$. By Lemma 4.5, we conclude that

$$
\operatorname{sign} \frac{\partial F}{\partial\left(g, g_{1}, \ldots, g_{n}\right)}=\operatorname{sign}\left((-\lambda)^{n+1} \operatorname{Hess}\left(\left.g\right|_{\{f=\delta\}}\right)\right) \quad \text { at } x \text {. }
$$

Applying Morse theory to $g$ on $\{f=\delta, g \geq 0\}$, we obtain that

$$
\chi\left(V_{\delta}(g \geq 0), V_{\delta}(g=0)\right)=\sum_{x \in X \cap V_{\delta}: g(x)>0} \operatorname{sign} \operatorname{Hess}\left(\left.g\right|_{\{f=\delta\}}\right)(x) .
$$

Applying Morse theory to $-g$ on $\{f=\delta, g \leq 0\}$, we also obtain that

$$
\chi\left(V_{\delta}(g \leq 0), V_{\delta}(g=0)\right)=(-1)^{n} \sum_{x \in X \cap V_{\delta}: g(x)<0} \operatorname{sign} \operatorname{Hess}\left(\left.g\right|_{\{f=\delta\}}\right)(x) .
$$

Taking the difference, we thus conclude that

$$
\chi\left(V_{\delta}(g \geq 0)\right)-\chi\left(V_{\delta}(g \leq 0)\right)=\sum_{x \in X \cap V_{\delta}, g(x) \neq 0} \operatorname{sign}(g(x))^{n+1} \operatorname{Hess}\left(\left.g\right|_{\{f=\delta\}}\right)(x) .
$$

By Lemma 4.3 (i), the condition $V_{\delta} \cap \Sigma(g)=\emptyset$ implies that 0 is a regular value of $\left.g\right|_{\delta_{\delta}}$, and we have

$$
\text { (4.8) }=\sum_{x \in X \cap V_{\delta}} \operatorname{sign}(g(x))^{n+1} \operatorname{Hess}\left(\left.g\right|_{\{f=\delta\}}\right)(x) .
$$


By Lemma 4.3 (ii), $\operatorname{sign}(f \lambda)=\operatorname{sign}(g)$ along each connected component of $X-\{0\}$, and we obtain that

$$
\begin{aligned}
(4.9) & =\sum_{x \in X \cap V_{\delta}} \operatorname{sign}(f(x) \lambda)^{n+1} \operatorname{Hess}\left(\left.g\right|_{\{f=\delta\}}\right)(x) \\
& =\operatorname{sign}(-\delta)^{n+1} \sum_{x \in X \cap V_{\delta}} \operatorname{sign}(-\lambda)^{n+1} \operatorname{Hess}\left(\left.g\right|_{\{f=\delta\}}\right)(x) \\
& =\operatorname{sign}(-\delta)^{n+1} \sum_{x \in X \cap V_{\delta}} \operatorname{sign} \frac{\partial F}{\partial\left(g, g_{1}, \ldots, g_{n}\right)}(x) \quad \text { by } \\
& =\operatorname{sign}(-\delta)^{n+1} \operatorname{deg} F,
\end{aligned}
$$

which implies the formula (4.2). The equality (4.3) follows from the deformation argument due to $[9, \S 11]$.

COROllary 4.6. Let $V$ be an analytic set of dimension $n+1$ defined near 0 in $\mathbf{R}^{m+n+1}$. Let $L$ be the nonsingular locus of $V \cap B_{\varepsilon}^{m+n+1}$ for small $\varepsilon>0$ and assume that $L$ is oriented. Let $g:\left(\mathbf{R}^{m+n+1}, 0\right) \rightarrow(\mathbf{R}, 0)$ be an analytic functiongerm. We assume that there are $C^{\infty}$-vector fields $v_{1}(x), \ldots, v_{n}(x)$ on $B_{\varepsilon}^{m+n+1}$ so that $v_{1}(x), \ldots, v_{n}(x)$ span the tangent space of $\left.g\right|_{L}$ at each $x \in L$ and the orientation of the level of $\left.g\right|_{L}$ there coincides with the orientation defined by $v_{1}(x), \ldots, v_{n}(x)$. Let $f:\left(\mathbf{R}^{m+n+1}, 0\right) \rightarrow(\mathbf{R}, 0)$ be an analytic function-germ. We assume that

$$
V_{\delta}=\left\{x \in V \cap B_{\varepsilon}: f(x)=\delta\right\}
$$

is nonsingular for a non-zero number $\delta$ which is sufficiently close to 0 . If $V_{\delta} \cap$ $\Sigma(g)=\emptyset$, the map-germ

$$
F:(L, 0) \rightarrow\left(\mathbf{R}^{n+1}, 0\right), \quad x \mapsto\left(f(x), v_{1} f(x), \ldots, v_{n} f(x)\right) .
$$

is finite and $\left.g\right|_{V_{\delta}}$ is Morse, then

$$
\begin{aligned}
\operatorname{deg}(F) & =\operatorname{sign}(-\delta)^{n+1}\left(\chi\left(V_{\delta}(g \leq 0)\right)-\chi\left(V_{\delta}(g \geq 0)\right)\right) \\
& =\operatorname{sign}(-\delta)^{n+1}\left(\chi\left(\bar{V}_{\operatorname{sign}(\delta)-}\right)-\chi\left(\bar{V}_{\operatorname{sign}(\delta)+}\right)\right)
\end{aligned}
$$

where $\bar{V}_{\operatorname{sign}(\delta) \pm}=\left\{x \in V \cap S_{\varepsilon}^{n}: \operatorname{sign}(\delta) f(x) \geq 0, \pm g(x) \geq 0\right\}$ for $0<\varepsilon \ll 1$.

Remark 4.7. We sketch how to find the formula (Theorem 4.3) in [2]. Let $\left(x_{0}, x_{1}, \ldots, x_{m+n+q}\right)$ denote a coordinate system of $\mathbf{R}^{m+n+q+1}$ at the origin. Let $n=1,3,7$, and let $m, q$ be non-negative integers. Let $f, g:\left(\mathbf{R}^{m+n+q+1}\right) \rightarrow(\mathbf{R}, 0)$ denote two analytic functions, and $h=\left(h_{1}, \ldots, h_{m}\right):\left(\mathbf{R}^{m+n+q+1}, 0\right) \rightarrow\left(\mathbf{R}^{m}, 0\right)$ a $C^{\infty}$-map. We assume that $g$ and $h$ do not depend on the last $q$ variables $x_{m+n+1}, \ldots, x_{m+n+q}$. Set $V=h^{-1}(0)$ and $L$ is the set of regular points of $V$ (i.e., $L=V-\Sigma(h))$. Since $L$ is orientable, we fix an orientation of $L$. Define vector fields $v_{1}, \ldots, v_{n+q}$ by 
$\left(v_{1}, \ldots, v_{n}\right)= \begin{cases}\left(v_{0,1}\right) & n=1, \\ \left(v_{0,1}+v_{2,3}, v_{0,2}-v_{1,3}, v_{0,3}+v_{1,2}\right) & n=3, \\ \left(v_{0,1}+v_{2,3}+v_{4,5}+v_{6,7}, v_{0,2}-v_{1,3}-v_{4,6}+v_{5,7},\right. & n=7, \\ v_{0,3}+v_{1,2}+v_{4,7}+v_{5,6}, v_{0,4}-v_{1,5}+v_{2,6}-v_{3,7}, & \\ v_{0,5}+v_{1,4}-v_{2,7}-v_{3,6}, v_{0,6}-v_{1,7}-v_{2,4}+v_{3,5}, & \\ \left.v_{0,7}+v_{1,6}+v_{2,5}+v_{3,4}\right) & \end{cases}$

where $v_{i, j}=\left|\begin{array}{ccccc}\partial_{x_{i}} & \partial_{x_{j}} & \partial_{x_{n+1}} & \cdots & \partial_{x_{n+m}} \\ g_{x_{i}} & g_{x_{j}} & g_{x_{n+1}} & \cdots & g_{x_{n+m}} \\ \left(h_{1}\right)_{x_{i}} & \left(h_{1}\right)_{x_{j}} & \left(h_{1}\right)_{x_{n+1}} & \cdots & \left(h_{1}\right)_{x_{n+m}} \\ \vdots & \vdots & \vdots & \ddots & \vdots \\ \left(h_{m}\right)_{x_{i}} & \left(h_{m}\right)_{x_{j}} & \left(h_{m}\right)_{x_{n+1}} & \cdots & \left(h_{m}\right)_{x_{n+m}}\end{array}\right|, \quad 0 \leq i<j \leq n$,

and $v_{n+1}=\partial_{x_{m+n+1}}, \ldots, v_{n+q}=\partial_{x_{m+n+q}}$. We remark that these vectors are the same as the vectors defined in subsection 2.1 when $(m, q)=(0,0)$. Consider the map

$$
F:(L, 0) \rightarrow \mathbf{R}^{n+q+1}, \quad x \mapsto\left(f, v_{1} f, \ldots, v_{n+q} f\right) .
$$

By (4.10), we obtain that

$$
\operatorname{deg} F= \pm\left(\chi\left(V_{\delta}(g \leq 0)\right)-\chi\left(V_{\delta}(g \geq 0)\right)\right.
$$

By the discussion in [3, §3], we obtain that $\operatorname{deg}(F)=\operatorname{deg}\left\{\left(F^{\prime}, h\right):\left(\mathbf{R}^{m+n+q+1}, 0\right) \rightarrow\right.$ $\left.\left(\mathbf{R}^{m+n+q+1}, 0\right)\right\}$ where $F^{\prime}$ is an extension of $F$ to $\left(\mathbf{R}^{m+n+q+1}, 0\right)$, and find Theorem 4.3 in [2].

Remark 4.8. Let $g:\left(\mathbf{R}^{n+1}, 0\right) \rightarrow(\mathbf{R}, 0)$ be a $C^{\infty}$-function and let $v_{1}, \ldots, v_{n}$ be vector fields on $\left(\mathbf{R}^{n+1}, 0\right)$ so that $\left\langle\nabla g, v_{i}\right\rangle=0, i=1, \ldots, n$. We denote by $\Sigma_{v}$ the set of points where $v_{1}, \ldots, v_{n}$ are linearly dependent. Let $f:\left(\mathbf{R}^{n+1}, 0\right) \rightarrow$ $(\mathbf{R}, 0)$ be a $C^{\infty}$-function so that $V_{\delta} \cap \Sigma(f)=\emptyset, \quad V_{\delta} \cap \Sigma(g)=\emptyset$ and $V_{\delta} \cap \Sigma_{v}=\emptyset$, where $V_{\delta}=\left\{x \in\left(\mathbf{R}^{n+1}, 0\right): f(x)=\delta\right\}$. If the map $F$ defined by (4.1) is finite and $\left.g\right|_{V_{\delta}}$ is Morse, then the same proof works and we obtain the formulas (4.2), (4.3). This observation is sometimes useful if we know $\Sigma_{v}$ explicitly.

Define

Here is an example that $\Sigma_{v}$ can be expressed explicitly. Set $p=1,3,8$.

$$
v_{i}= \begin{cases}\text { the same as in subsection } 2.1 \text { replacing } n \text { by } p \text { there } & i=1, \ldots, p \\ g_{x_{i}} \nabla g-\|\nabla g\| \partial_{x_{i}} & i=p+1, \ldots, n\end{cases}
$$

Then we obtain $\Sigma_{v}=\left\{g_{x_{0}}=\cdots=g_{x_{p}}=0\right\}$. Suppose that $g(x)=\sum_{i=0}^{n} x_{i}^{2}$. Then $\Sigma_{v}=\left\{x_{0}=\cdots=x_{p}=0\right\}$. If $f:\left(\mathbf{R}^{n+1}, 0\right) \rightarrow(\mathbf{R}, 0)$ defines an isolated singularity with $f\left(\Sigma_{v}\right)=0$, and the map $F$ defined by (4.1) is finite, then we obtain that

$$
\operatorname{deg} F=(-1)^{n} \chi\left\{x \in S_{\varepsilon}^{n}: f(x) \geq 0\right\} .
$$

To state a global consequence of our theorem, we introduce the following 
Definition 4.9. Let $M$ be a $C^{\infty}$-manifold and let $\varphi: M \rightarrow \mathbf{R}$ be a $C^{\infty}$ function. We say that Morse theory is applicable to $\varphi$ on the closed interval $[a, b]$ if the following two conditions hold.

(1) $\varphi$ has at most finitely many critical points in $\varphi^{-1}[a, b]$, and all critical points are Morse singularities, that is, the Hessian determinant $\operatorname{Hess}(\varphi)(x)$ of $\varphi$ is non zero at each critical point $x$.

(2) there is "no surgery at infinity" on $[a, b]$, which means that $\{x \in M$ : $\varphi(x) \leq c-\varepsilon\}$ and $\{x \in M: \varphi(x) \leq c+\varepsilon\}$ are diffeomorphic each other for sufficiently small $\varepsilon>0$ when $c$ is not a critical value of $\varphi$ with $c \in[a, b]$.

THEOREM 4.10. Let $L$ be a real analytic manifold of dimension $n+1$ and let $f, g: L \rightarrow \mathbf{R}$ be analytic functions. We assume that $V_{\delta}=\{x \in L: f(x)=\delta\}$ is nonsingular for a non-zero number $\delta$ with $0<|\delta| \ll 1$ and Morse theory is applicable for $\left.g\right|_{V_{\delta}}$ on $\left[b_{0}, b_{k}\right]$. We assume that $g$ satisfies Condition $(P)$, and that the map

$$
F: L \rightarrow \mathbf{R}^{n+1}, \quad x \mapsto\left(f(x), v_{1} f(x), \ldots, v_{n} f(x)\right),
$$

is finite. We set $F^{-1}(0)=\left\{P_{1}, \ldots, P_{k}\right\}$ and $c_{i}=g\left(P_{i}\right)$ for $i=1, \ldots, k$, and assume that $b_{0}<c_{1}<c_{2}<\cdots<c_{k}<b_{k}$. Taking $b_{i}$ with $c_{i}<b_{i}<c_{i+1}$ for $i=$ $1, \ldots, k-1$, we have

$$
\operatorname{deg}(F)=\operatorname{sign}(-\delta)^{n+1} \sum_{i=1}^{k}\left(\chi\left(V_{\delta}\left(b_{i-1} \leq g \leq c_{i}\right)\right)-\chi\left(V_{\delta}\left(c_{i} \leq g \leq b_{i}\right)\right)\right)
$$

Moreover, if $n$ is odd, we have

$$
\operatorname{deg}(F)=\chi\left(V_{\delta}\left(b_{0} \leq g \leq b_{k}\right), V_{\delta}\left(g=b_{0}\right)\right) .
$$

Proof. By Theorem 4.1, we obtain that

$$
\operatorname{deg}(F) \text { at } P_{i}=\operatorname{sign}(-\delta)^{n+1}\left(\chi\left(V_{\delta}\left(b_{i-1} \leq g \leq c_{i}\right)\right)-\chi\left(V_{\delta}\left(c_{i} \leq g \leq b_{i}\right)\right)\right) .
$$

This implies (4.11). When $n$ is odd, the proof of Theorem 4.1 implies

$$
\operatorname{deg}(F)=\sum_{x \in X \cap V_{\delta}} \operatorname{Hess}\left(\left.g\right|_{V_{\delta}}\right)(x)
$$

and the right hand side is equals to

$$
\chi\left(V_{\delta}\left(b_{0} \geq g \geq b_{k}\right), V_{\delta}\left(g=b_{0}\right)\right),
$$

which completes the proof of (4.12).

\section{Mapping degree of $\bar{p}([d g],[d f])$}

We denote by $\pi: \mathbf{R}^{n+1}-\{0\} \rightarrow S^{n}$ the projection defined by $x \mapsto x /\|x\|$. Let $f:\left(\mathbf{R}^{n+1}, 0\right) \rightarrow(\mathbf{R}, 0)$ be a $C^{\infty}$-function-germs. We define a map $[d f]$ : 
$S_{\varepsilon}^{n} \rightarrow S^{n}$ by $x \mapsto \pi \circ d f(x)$ where $S_{\varepsilon}^{n}$ denotes the $n$-sphere centered at 0 with radius $\varepsilon$ and $S^{n}$ denotes the unit sphere centered at 0 . Suggested by Remark 2.1, we are interesting in the following: Let $f, g:\left(\mathbf{R}^{n+1}, 0\right) \rightarrow(\mathbf{R}, 0)$ be two $C^{\infty}$ function-germs. We consider a smooth map $p: \mathbf{R}^{n+1} \times \mathbf{R}^{n+1} \rightarrow \mathbf{R}^{n+1}$ and set $\tilde{Z}=p^{-1}(0)$. We investigate the mapping degree of the map

$$
\bar{p}([d g],[d f]): S_{\varepsilon}^{n} \rightarrow S^{n}, \quad x \mapsto \pi \circ p([d g](x),[d f](x))
$$

when $Z:=\tilde{Z} \cap\left(S^{n} \times S^{n}\right)$ is empty.

LEMMA 5.1. Let $M$ be an oriented manifold of dimension $\geq n$ and let $\omega$ be the volume form of the sphere $S^{n}$ so that $\int_{S^{n}} \omega=1$. We consider a $C^{\infty}$-map $f: M \rightarrow S^{n}$. Then $\operatorname{deg}\left(\left.f\right|_{X}\right)=\int_{X} f^{*} \omega$ for any oriented $n$-cycle $X$ of $M$ so that $\left.f\right|_{X}$ is proper and finite.

The proof is similar to the proof of Theorem 12 in [10, Chapter 8].

Proof. Let $y$ be a regular value of $\left.f\right|_{X}$ and let $U$ be an open neighborhood of $y$. Let $\omega^{\prime}$ be an $n$-form of $S^{n}$ which is cohomologous to $\omega$ and $\operatorname{supp}\left(\omega^{\prime}\right) \subset U$. Let $\left\{x_{1}, \ldots, x_{k}\right\}$ be the preimage of $y$. Choosing $U$ small we may assume that $\left(\left.f\right|_{X}\right)^{-1}(U)=U_{1} \cup \cdots \cup U_{k}$ where each $U_{i}$ is an open neighborhood of $x_{i}$ in $X$ and each $U_{i}$ is diffeomorphic to $U$. Then we have $\int_{U_{i}}\left(\left.f\right|_{X}\right)^{*} \omega^{\prime}= \pm \int_{U} \omega^{\prime}= \pm 1$ where the sign is $+($ resp. -$)$ when $\left.f\right|_{U_{i}}$ is orientation preserving (resp. reversing). Thus we have

$$
\operatorname{deg}\left(\left.f\right|_{X}\right)=\sum_{i=1}^{k} \int_{U_{i}}\left(\left.f\right|_{X}\right)^{*} \omega^{\prime}=\int_{X}\left(\left.f\right|_{X}\right)^{*} \omega^{\prime}=\int_{X}\left(\left.f\right|_{X}\right)^{*} \omega=\int_{X} f^{*} \omega
$$

and this completes the proof.

Let $\boldsymbol{e}_{i}, i=0,1, \ldots, n$, denote the unit vector $(0, \ldots, \stackrel{i+1}{1}, \ldots, 0)$ in $\mathbf{R}^{n+1}$. We investigate when $Z$ is empty. When $Z=\emptyset$, we can consider the following map:

$$
\bar{p}: S^{n} \times S^{n} \rightarrow S^{n}, \quad(x, y) \mapsto \pi \circ p(x, y) .
$$

We define the class of $\bar{p}$, denoted by $h(\bar{p})$, the image of the fundamental class of $S^{n}$ by the map

$$
H^{n}\left(S^{n} ; \mathbf{Z}\right) \stackrel{\bar{p}^{*}}{\rightarrow} H^{n}\left(S^{n} \times S^{n} ; \mathbf{Z}\right)=\mathbf{Z}^{2},
$$

where the last equality presents the natural identification between the cohomology group $H^{n}\left(S^{n} \times S^{n} ; \mathbf{Z}\right)$ and the free $\mathbf{Z}$-module generated by the cohomology classes corresponding to $S^{n} \times \boldsymbol{e}_{0}$ and $\boldsymbol{e}_{0} \times S^{n}$.

Proposition 5.2. There is a $C^{\infty}$-map $\bar{p}: S^{n} \times S^{n} \rightarrow S^{n}$ so that $h(\bar{p})=$ $\left(k_{1}, k_{2}\right)$ if and only if one of the following conditions holds.

- $n=1,3,7$.

- $n$ is odd $, n \neq 1,3,7$, and $k_{1} k_{2} \equiv 0(\bmod 2)$.

- $n$ is even, and $k_{1} k_{2}=0$. 
Proof. Assume first that $n$ is even. Let $\omega$ denote the volume form of $S^{n}$. Let $p_{i}: S^{n} \times S^{n} \rightarrow S^{n}, i=1,2$, denote the $i$-th projection. We remark that $\bar{p}^{*} \omega$ is cohomologous to $k_{1}\left(p_{1}\right)^{*} \omega+k_{2}\left(p_{2}\right)^{*} \omega$. The assertion comes from the following:

$$
\begin{aligned}
0=\left(\bar{p}^{*} \omega\right) \wedge\left(\bar{p}^{*} \omega\right) & =\left(k_{1}\left(p_{1}\right)^{*} \omega+k_{2}\left(p_{2}\right)^{*} \omega\right) \wedge\left(k_{1}\left(p_{1}\right)^{*} \omega+k_{2}\left(p_{2}\right)^{*} \omega\right) \\
& =2 k_{1} k_{2}\left(p_{1}\right)^{*} \omega \wedge\left(p_{2}\right)^{*} \omega .
\end{aligned}
$$

We next consider the case that $n$ is odd. Let $f_{i}: S^{n} \rightarrow S^{n}$ be a $C^{\infty}$-map of degree $k_{i}$. We remark that their homotopy classes is $k_{i} l_{n}$ where $l_{n}$ is the identity map of $S^{n}$. It is enough to determine all $\left(k_{1}, k_{2}\right)$ so that the Whitehead product $\left[k_{1} l_{n}, k_{2} l_{n}\right]=k_{1} k_{2}\left[l_{n}, l_{n}\right]$ vanishes. By the theorem of J. Adams [1, Theorem 1.1.1], $\left[l_{n}, l_{n}\right]=0$ if and only if $n=1,3,7$. This implies the second assertion. Since $\left[l_{n}, l_{n}\right]$ is of order 2 when $n \neq 1,3,7$, we obtain the last assertion.

When $n=1,3,7$, and a map $\bar{p}$ with $\left(k_{1}, k_{2}\right)=(1,1)$, is induced by the product of complex, quotanion, Cayley numbers respectively.

When $n=1$, we identify $\mathbf{R}^{2}$ with $\mathbf{C}$ by $(x, y) \mapsto z=x+y i$. The map $p_{k_{1}, k_{2}}: \mathbf{R}^{2} \times \mathbf{R}^{2} \rightarrow \mathbf{R}^{2}$ defined by $\left(z_{1}, z_{2}\right) \mapsto z_{1}^{k_{1}} z_{2}^{k_{2}}$ represents a map which class is $\left(k_{1}, k_{2}\right)$. Remarking $z^{-1}=\bar{z}$ on $S^{1}$, we see all the classes $\left(k_{1}, k_{2}\right)$ are represented by polynomial maps.

When $n$ is odd, a map $S^{n} \times S^{n} \rightarrow S^{n}$ with $\left(k_{1}, k_{2}\right)=(1-k, k)$ is represented by the following way: Take $x, y \in S^{n}$, and consider the great circle containing $x, y$. The image of $(x, y)$ is $z$ in the great circle defined by $\measuredangle x 0 z=k \measuredangle x 0 y$ described in the following picture in the case $k=-2$.

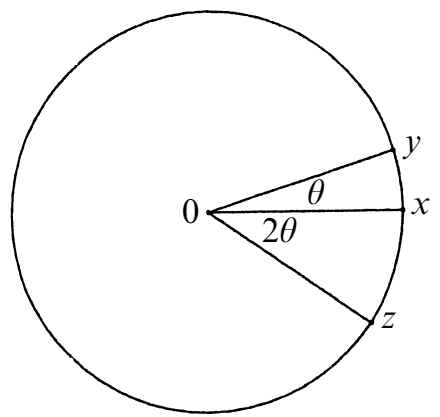

An explicit formula for this map is described by the following: For $x, y \in S^{n}$, we set $z=p_{k}(u, v) x+q_{k}(u, v)(y-u x)$ where $u=\langle x, y\rangle, v=|y-u x|$. Here $p_{k}(u, v)$ and $q_{k}(u, v)$ denote real polynomials defined by $(u+v i)^{k}=p_{k}(u, v)+q_{k}(u, v) v \boldsymbol{i}$.

Proposition 5.3. Let $\bar{p}: S^{n} \times S^{n} \rightarrow S^{n}$ be a $C^{\infty}$-map with $h(\bar{p})=\left(k_{1}, k_{2}\right)$, and let $f_{i}: S^{n} \rightarrow S^{n}, i=1,2$, be two $C^{\infty}$-maps. We define a map by 


$$
f:=\bar{p}\left(f_{1}, f_{2}\right): S^{n} \rightarrow S^{n}, \quad x \mapsto \bar{p}\left(f_{1}(x), f_{2}(x)\right) .
$$

Then we have $\operatorname{deg}(f)=k_{1} \operatorname{deg}\left(f_{1}\right)+k_{2} \operatorname{deg}\left(f_{2}\right)$.

Proof. Let $\omega$ denote the volume form of $S^{n}$ with $\int_{S^{n}} \omega=1$. Let $p_{i}$ : $S^{n} \times S^{n} \rightarrow S^{n}, \quad i=1,2$, denote the $i$-th projection. We remark that $\bar{p}^{*} \omega$ is cohomologous to $k_{1}\left(p_{1}\right)^{*} \omega+k_{2}\left(p_{2}\right)^{*} \omega$. Then we have $\operatorname{deg}(f)=\int_{S^{n}} f^{*} \omega=$ $\int_{S^{n}}\left(k_{1}\left(p_{1}\right)^{*} \omega+k_{2}\left(p_{2}\right)^{*} \omega\right)=k_{1} \operatorname{deg}\left(f_{1}\right)+k_{2} \operatorname{deg}\left(f_{2}\right)$.

\title{
REFERENCES
}

[1] J. F. Adams, On the non-existence of elements of Hopf invariant one, Ann. of Math. 72 (1960), 20-104.

[2] N. Dutertre, Degree formula for a topological invariant of bifurcations of function-germs, Kodai Math. J. 23 (2001), 442-461.

[3] T. FuKUI, An algebraic formula for a topological invariant of bifurcation of 1-parameter family of function-germ, Stratifications, singularities and differential equations II, Stratifications and topology of singular spaces (D. Trotman \& L. Wilson eds.), Travaux en cours 55, Hermann, 1997, 45-54.

[ 4 ] T. FukUI, Mapping degree formula for 2-parameter bifurcation of function-germs, Topology 32 (1993), 567-571.

[5] S. Hu, Homotopy theory, Pure and applied mathematics VIII, Academic Press, 1959.

[6] D. Husemoller, Fiber bundles, 3rd ed., Graduate texts in mathematics 20, Springer-Verleg, 1993.

[ 7 ] G. N. Khimshiashvili, On the local degree of a smooth mapping (in Russian), Bull. Acad. Sci. Gerogian SSR 85 (1977), 309-312.

[8] J. Milnor, Topology from the differentiable viewpoint, The University Press of Virginia, Charlottesville, 1965.

[9] J. Milnor, Singular points of complex hypersurfaces, Annals of Mathematical Studies 61, Princiton University Press, 1968.

[10] M. SpIVAK, A comprehensive introduction to differential geometry, 2nd ed., I, Publish or Perish, Inc., Wilmington, Del., 1979.

\author{
Department of Mathematics \\ FACUlTY OF SCIENCE \\ SAITAMA UNIVERSITY \\ 255 Shimo-OKubo, SaKura-KU \\ SAITAMA $338-8570$ \\ JAPAN \\ E-mail: tfukui@rimath.saitama-u.ac.jp \\ Department of Mathematics \\ UNIVERSITY OF TORONTO \\ Toronto, ON M5S 3G3 \\ CANADA \\ E-mail: askold@math.toronto.edu
}

